\title{
CANCER OF THE BREAST *
}

\author{
BY
}

\author{
CECIL ROWNTREE, F.R.C.S. \\ Senior Surgeon, Royal Cancer Hospital, London
}

It is natural that those of us who spend a large part of our lives in the Royal Cancer Hospital should regard cancer of the breast as a very common disease; and so it is within the walls of that hospital, as will be appreciated when I say that about one hundred cases come for treatment every year.

But of course we cast our net wide, and in order to get a true picture of the real frequency of the disease it is necessary to think statistically for a moment and to remember that while there are over 33,500 doctors in England, there would appear to be not more than 6,500 fresh cases of cancer of the breast each year. Equally divided among us all, this number gives only a fifth of a case apiece. It follows, therefore, that cancer of the breast is not really common enough to enable many of us to have enough personal experience to assess the value of different methods of treatment. We are all apt to be unduly influenced by one case-often the last one seenand all sorts of fallacies result. One practitioner who has recently had one or two good results following upon operative treatment may think it is all that can be desired; while another man in the next street, having recently seen a case of recurrence, thinks all treatment useless and the disease hardly worth treating.

As is usual in so many other problems, the truth lies somewhere in between, and I want to show both sides of the picture. On the one hand we see grounds for the greatest optimism, and on the other bitter disappointments and heart-breaking failures. But in spite of these variations cancer of the breast is a disease which, in the aggregate, does notably maintain a very steady average picture.

\section{Some Figures}

Let me give you some figures. Consider, for instance, cases of cancer of the breast in which no active surgical or radiological treatment has been employed. Of course we cannot get such figures now, as all cases are treated by one or other of these methods; but there are plenty of statistics worked out by an earlier generation, and they show that on the average a woman with cancer of the breast lived three and a half years when no treatment of any kind was carried out.

This is a very important figure, for it is upon this datum line that the average success or otherwise of any method of treatment must be judged. And what do we find? The statistics so carefully collated and analysed by the Ministry of Health show that operative treatment, even at its very worst (for its figures only relate to patients that died of recurrence after operation) adds three years to this average; so that women with cancer of the breast who come for surgical treatment may

* A lecture delivered at the annual meeting of the Oxford Medical Society on November 13th, 1936. expect to live an average of six and a half years from the first onset of the disease. This is the worst. What is the best that can be expected?

\section{History of Surgical Treatment}

But before discussing after-results, let me recall the history of the surgical treatment of breast cancer. It is a long story in point of time, for it goes back to the dim ages at the dawn of surgical history, when the cancerous breast was excised by the crude methods then available. And for century after century the same primitive local excision continued to be practised until the coming of Lister put surgery on a different basis. Surgeons were no longer compelled to make their operations as simple as possible owing to the certainty of infection of the wound; they were now able to plan their incisions, fashion their flaps, dissect out the muscles, and, indeed, fit their operations to meet the conditions imposed by the disease. Pathological anatomy began to play its part in dictating an operative scheme, and surgeons ceased to be artisans and became pathologists as well.

In 1894 Halsted gave us what is to all intents and purposes the modern operation for amputation of the breast. His operation was based on the pathological anatomy of the cancerous breast and its extensions, and upon observation of the most usual sites for secondary nodules and recurrences. It at once put the outlook for these cases on a different footing, but in spite of great improvement in results critical study of the after-history of the cases revealed a disappointing percentage of failures.

The next great step forward was Sampson Handley's enunciation of the principle of lymphatic permeation and the planning of various modifications of the Halsted operation, with a view to meeting the situation indicated by his theory. Very briefly, the salient points were a less wide removal of the skin (which was a great blessing both to the patient and to the surgeon) and a much wider removal of the deep fascia in which the vulnerable lymphatics lay. Although there are many who do not accept Handley's views in their entirety, yet one notices that it is Handley's operation that is the standard technique employed in every clinic in the surgically civilized world, and this operation may be said to represent the zenith of the purely surgical treatment of cancer of the breast.

It has often been said of the breast, as of other organs, that operative treatment has reached its limit. Although it is true that a limit has probably been reached as to the amount of tissue removed (for some surgeons say they remove everything but the bones), yet as to the method of its removal, who can say what the future may have in store for us? We had an indication of the sort of development that may take place when the use of the 
diathermy knife was introduced for removing the breast. This was a fundamental change in technique which had its day, and in fact still has its day in certain chosen cases, more especially in advanced or ulcerated cases, when local infection is a special risk.

\section{Points in Amputation of Breast}

I am not going to describe the technical minutiae of amputation of the breast, but I would like to emphasize certain points of importance that are sometimes overlooked.

The first is the value of a routine radiological examination, especially of the spine and thorax, in order to eliminate these not very rare cases in which an unsuspected bone metastasis has already established itself. During the actual operation I would lay chief stress on the importance of avoiding cooling of the large surface of the wound, and of making sure that one's assistant is not leaning too heavily upon the other side of the thorax. It is important, too, that tension on the stitches should be avoided at all costs in order to prevent that aseptic sloughing of the flaps that is one of the most troublesome complications of this operation. The only special point in the immediate post-operative treatment is to avoid the painful and trying method of fixing the arm at right angles to the body, which is sometimes employed in the mistaken belief that it will prevent post-operative stiffness. Shock and loss of blocd are, of course, to be guarded against and to be remedied, either by blood transfusion or by continuous intravenous glucose saline.

It is hardly possible to say that any operaticn can be expected to be entirely free from mortality, but the mortality from amputation of the breast should be, and at the Royal Cancer Hospital is, almost negligible. Personally I have been lucky enough to lose only one case, and in this case death took place from that still mysterious disaster-pulmonary embolism.

Post-operative morbidity also is extremely low. Infection of the wound is rare, and the stiffness of the shoulder so greatly feared by the earlier operators is a complication that we never meet with now. Indeed, it is amazing to think that so destructive and mutilating an operation should be followed by so little disability. The removal of the greater portion of the pectoral muscle and the whole of the pectoralis minor as well, does not appear to prevent quite respectable performances on the golf course or the tennis court, to say nothing of a hard day's work in the house.

\section{Results of the Operation}

What are the results that we may expect after such an operation as I have described, undertaken by an expert operator under ideal conditions, in an otherwise healthy woman? We know only too well ; for the best statistics, furnished by the best surgeons in the world, show that less than half their patients will be free from any signs of the disease at the end of five years, even when the growth is in its earliest stage, apparently confined to the breast itself, and where no evidence of invasion of the glands can be detected on the most careful microscopical examination. In cases in which the disease is more advanced-where, for instance, the axillary glands are palpable on examination and found to be invaded on nathological investigation-the figure drops to something like 20 per cent. And when our cases are later still and have reached the stage in which the most casual glance is enough to determine the diagnosis, then the survival rate drops to almost nothing.

Do not misunderstand me, for there are, of ccurse, brilliant exceptions, and quite advanced cases are some- times operated upon so successfully that the patients come to forget that they have ever been ill. But unhappily the converse of the picture is also true, and the earliest and apparently most favourable cases will sometimes develop recurrence within a few months. We surgeons have to live a long time before we have lived long enough to have had the unhappy experience of seeing many of our best efforts and our most favourable cases disappoint us in this grim fashion. But we all come to it in time, and we must face the fact that the last few years have not seen any marked improvement, or indeed any improvement at all, worthy of the name, in the results from purely surgical treatment. It is natural, therefore, that we should turn to other methods in the hope of some support for our surgical attack.

\section{$\mathrm{X}$ Rays as an Adjunct to Operation}

We have two important agents at our disposal- $x$ rays and radium. As in $x$ rays we have an agent which is easily applied, is free-or almost free-from danger, and is available at a good many centres, the custom has gradually developed of giving patients subjected to surgical operation a course of $x$-ray therapy as well. There is a good deal of controversy as to whether it should be administered before or after the operation, and there is much to be said in support of either method. Those who favour prior $x$-ray treatment point out that the activity of the tumour may be diminished; that sparsely scattered outlying cells may be destroyed; and that the danger of cell dissemination during the subsequent operation will be reduced to a minimum. Against this is urged, first, the delay in operative treatment that inevitably results from it; secondly, the possibility that the operaticn may be more difficult and healing more protracted (although personally I think that this objection is without much weight); and, thirdly, that the tumour may be shown by the operation not to be malignant at all.

But to my mind the real objecticn to the use of deep therapy before operation is psychological. In all conscience it is sometimes difficult enough to persuade a patient to submit to amputation of her breast; it is still more difficult if it is explained that she must first undergo a course of three or four weeks' treatment before the moment for operation arrives. My own feeling, therefore, is that if $x$-ray treatment as an adjunct to surgical operation is carried out at all, it should be done after the operation, and there seems to be no objection to starting it within a very few days of the healing of the wound.

There would appear to be good ground for claiming that pre-operative or post-operative deep therapy has already effected some improvement in results, although it is too soon yet to say whether or no an intensive trial of this method is going to make a really great improvement in our ultimate statistics. But its application is so rational and so safe in skilled hands; the power and scope of $x$-ray apparatus is improving so rapidly; and, moreover, it is so urgent that something be done to better cur results that $I$ hold the view that all our cases of radical amputation should receive it.

\section{Radium Therapy}

The other great adjunct to surgery is radium, and in its use for cancer of the breast Handley again deserves an honoured place among the pioneers. Long years ago he pointed out that one of the difficulties of surgical operation was the impossibility of following the lymphatic tracks that wind through the upper intercostal spaces to reach the thorax, and he endeavoured to overcome this short- 
coming of the radical operation by implanting tubes of radium into the vulnerable intercostal spaces.

Then we pass to another stage. Regaud's work in establishing the value of the long-continued application of small quantities of radium, widely scattered through the affected area, gave a new impetus to the treatment of cancer of the breast by radium therapy. A large number of advanced cases, unsuitable for surgical operation, were subjected to this new form of treatment. The results in many cases were dramatic-so dramatic that it was apparent that a new method had evolved which was likely to have far-reaching consequences. And so it had, for within a comparatively short time Keynes at St. Bartholomew's Hospital began to undertake the treatment of early operable cases of cancer of the breast by interstitial radium. And Keynes was soon followed by a good many other surgeons, who felt sufficient dissatisfaction with purely operative methods to lead them to embark upon this new experiment. It was a venture for which there was much support to be found, not only in the results obtained in such sites as the lip, tongue, and cervix uteri, but also in the highly satisfactorythough only temporary-benefits resulting from the radium treatment of even the most advanced cases of cancer of the breast. The accessibility of the breast, its ease of observation, and the large number of cases available for comparison, coupled with the extreme willingness of the patients to submit to this new form of treatment, all combined to give a powerful impetus to the method. This resulted in a large number of cases being dealt with on these lines in London, New York, and other great centres. We naturally played our part at the Royal Cancer Hospital in seeking opportunities for a test of what we may call the radical radium treatment of early cases.

\section{Radical Radium Treatment of Early Cases}

Very briefly, the technique employed consisted in the implantation of radium needles of various lengths in such a way as completely to penetrate and surround the primary focus and the areas of probable lymphatic spread. At the Royal Cancer Hospital we used platinum needles containing radium salt. In New York they rather specialized in radon, contained in gold capillary tubes, which have the great advantage that they can be obtained in almost unlimited quantity and in sections of great length.

These are all matters of detail and of no fundamental importance, providing the quantity of radium or radon is sufficient, the duration of dosage long enough, and the spacing and distribution suitably planned to meet the circumstances of the case.

What are the results to date? It is early days to give carefully analysed figures of the ultimate or even interim fate of our patients, for this is a matter of years. But it is fair to say that on the whole the results support the view that this method of treatment does give results which are at least comparable with those obtained by operative treatment. But statistics alone, until they become so overwhelming in quantity and quality as to prove a point beyond all discussion, are not always sufficient by themselves to determine current practice. And it becomes pretty clear what the general feeling of most of the surgeons at the Royal Cancer Hospital is when I tell you that the proportion of radical amputations carried out there has increased in the last year or so. The pendulum is swinging back, or has swung back, to the operative treatment of early cases.

Of course, as in all experiments that show some measure of success, one tends to be too optimistic, and I think now that we were all perhaps a little too hopeful that a method had been achieved which would do as much or more for our patients than operative treatment, and would at the same time spare them the distress and mutilation of amputation.

\section{Difficulties of Radical Radium Therapy}

Radical radium treatment did not prove quite as simple as we had hoped. In the first place, the amount of work involved in recording, sketching, and following up the cases enormously added to the clerical work of the hospital, and of course to the expense. We were faced with the constant difficulties occasioned by stupidity on the part of patients who could not or would not understand the necessity for a careful follow-up, and perhaps the need for further treatment. But above all, we were faced with new conditions not hitherto experienced: extensive radium burns and their explanation and treatment; and in particular the difficulty caused by the fact that some of the tumour masses failed to resolve in spite of intensive treatment. And what, then, was to be done with them? Further long exposures, and the possibility of really serious burns? Or surgical removal and invalidation of the whole experiment? Some of the masses were removed, of course, and found to contain living cancer cells. But the fate of similar masses that had not been removed served strongly to suggest that the cancer cells in question had been reduced to a condition of inactivity, although no man could say how long such inactivity might be expected to persist.

Some of these difficulties are of a nature that time, increased facilities, better team work, and a clearer comprehension of their origin will in due course adjust for us. But some of them are fundamental to the method, and I see no way of getting over them. I have some doubt as to whether any successful plan of campaign of this sort will ever be evolved which will give us what we scarcely have the right to ask of any method. For we expect our method to deal with a disease of which the nature is unknown, its precise method of extension only suspected, and its growing edge an indeterminate nebulous zone which in one case may be confined to the breast, and in the next (to all appearances quite identical) may already have extended within the chest itself. It is difficult to imagine any possible distribution of radium which will overcome these obstacles. My view, therefore, is that in the present state of our knowledge all early and favourable cases should be subjected to radical operation.

But what are we to do if during the course of an operation for what looks like an early favourable case we discover that the disease is more extensive than anticipated, and the glands obviously and grossly invaded up to the apex of the axilla? In these cases I always remember that I am faced with the possibility not only of failing to do good but of really doing harm. What surgeon is there who cannot recall instances when a radical operation in an advanced case of this kind has been followed by widespread local dissemination, extensive cancerous infiltration of the skin and subcutaneous tissues, and early onset of that most distressing complication of all-the mammoth arm? Accordingly, when I am faced with this situation (and this is where I perhaps differ from some of my surgical colleagues) I am tempted to remove only the grosser part of the disease, leaving a respectable margin of healthy tissue to cover over what I have had perforce to leave behind, in the hope that other treatment may succeed in doing what $I$ have failed to do with the knife. In such cases $I$ believe that radium 
is of great assistance, and I subject them either at the time, or later, to an intensive barrage of radium needles.

\section{The Value of Radium}

I must not leave you with the view that I am a pessimist about the value of radium. Indeed, anyone who has worked in cancer for as long as I have can be nothing but an optimist when he compares the composite picture cf cancer treatment and its results at the present time with what they were ten years ago. The whole aspect of affairs has changed. Tongue, mouth, pharynx, uterus, and breast have all shared in the general amelioration, some more than others, some less; and I should emphasize that it is only in so far as the treatment of early breast cancer is concerned (where our hopes were high) that radium has disappointed me. But it is only a relative disappointment. Radium has furnished us with the most valuable method of special treatment of cancer of the breast at our disposal, and it would be utterly impossible to do without it for our advanced cases, or for those which for any other reason are inoperable.

Deep therapy is catching it up, but it has not caught up yet. However, my radiological colleagues tell me that the time is coming when they will be able to provide us with an $x$-ray beam comparable in the shortness of its wavelength with the gamma rays of radium, but delivered with an intensity and in such a quantity as no source of radium supply is ever likely to attain, unless, of course, radium becomes available in almost unthinkable quantities. When that time comes, or when $x$ rays do become equivalent in power to the gamma rays of radium, a new and happier chapter in the history of cancer of the breast will open, and we surgeons once again will have to pause and reconsider our position.

\section{Early Diagnosis}

You will observe that I have said nothing yet as to the diagnosis of cancer of the breast, and after all, it is the most important preliminary to treatment, whatever that may be. I think the most vital point about diagnosis is to remember that it is daily becoming more difficult. The influence of propaganda has been so great and so much has been written in the lay and medical press as to the importance of obtaining treatment at the earliest possible moment, that our cases are coming to us at a very much earlier stage than they ever have before. The diagnosis of a well-developed cancer of the breast is easy; but the diagnosis of a tiny lump no bigger than a pea is sometimes quite impossible until it has been removed. One sometimes hears an argument as to whether there is any danger in cutting into a breast tumour for verification of its nature. I believe there is danger; and I think that in those cases, increasing as they are in number, in which it is impossible to feel confident as to the nature of a suspicious nodule, it should be completely removed, with a fairly wide margin of healthy tissue round it. Then, and only then, should it be bisected, when its nature will almost always be at once apparent to the naked eye.

It is interesting to note that a special investigation carried out by the Imperial Cancer Research Fund, on "the importance of microscopical investigation," showed that the diagnosis of cancer of the breast was less often incorrect than that of cancer in any other part of the body.

It is obvious, I suppose, that the removal of these doubtful tumours of the breast should only be undertaken by surgeons who have had that long practical training in laboratory and operating theatre which gives them the ability to recognize the nature of the tumour they are dealing with and the capacity for coping with unexpected situations.

Frozen sections are sometimes employed as an aid to diagnosis in the operating theatre, and they are of the utmost value in certain sites. But I feel that when an experienced practical surgeon is in doubt about the nakedeye appearance of a breast. tumour the frozen section is just as likely to be as difficult of interpretation as the gross pathological picture.

One other matter that I must not omit. Do benign tumours of the breast ever become malignant? I think not; but it is clearly not a question capable of final proof. If we see a little tumour to-day and tell the patient "I think that is an adenoma, and therefore do not bother about it," and she comes back a year hence with carcinoma, that does not prove that a simple tumcur has become malignant; it merely shows that we have been most unwise and have overlooked a carcinoma in its very early stage. Who can say with positive certainty that a lump is simple or malignant until it has been removed? I cannot, although I can generally venture a pretty shrewd guess, and I am quite often right; but I am also often wrong. There can be no excuse for that pernicious practice, the results of which we still sometimes see, of telling a woman not to bother about a lump in the breast unless, or until, it gives trouble. When it does give trouble it is trouble indeed.

\section{A Working Plan}

In conclusion, I should like to feel in a position to give you absolutely emphatic, positive guidance as to what you ought to advise in breast cases that come to your notice; but I do not think that the position is such, especially at the present moment, as to lend itself to emphatic pronouncements. I have, however, developed for myself a fairly definite working scheme, based upon a long experience and upon much thought about my own work and observation of that of others.

My attitude is that all operable cases of cancer of the breast should be subjected to radical amputation, followed by a course of prophylactic high-voltage and short-wayelength $x$-ray therapy. But, you will say, what constitutes an operable case? And here we are on debatable ground. Let me first of all tell you of the cases that I regard as surgically inoperable. I put first those with widespread extension of the growth; or those with secondary skin nodules, or indeed one nodule outside the breast area; or wide ulceration or oedema of the skin ; or enlargement of the supraclavicular glands; or the presence of a distant metastasis; or involvement of both breasts; or enlarged fixed glands in the axilla.

But there are other factors, too, which render a case inoperable. I may call them medical. And among these I include pregnancy and lactation; serious intercurrent disease ; extreme youth (for example, cancer occurring under the age of 25 , as in my experience surgical treatment here is always hopeless); or in such extreme old age that the general expectation of life is less than the average duration of the disease.

If we exclude all these, it leaves us with thcse patients who are not too young nor yet too old; whose tumour is confined to the breast itself and not adherent to the chest walls; in whom the axillary glands have not become grossly enlarged, and where no metastasis can be discovered.

Of course old age in itself is not a bar to operation, providing the general condition of the patient is satisfactory. I operated upon one patient of 82 , who survived 
ten years. Nor is the mere size of the primary growth of first importance, as long as the extension is outward as it were, and not inward towards the chest wall.

So far the indications are clear and our course is easy. But what are we to do for patients not suitable for radical operation? We will consider first those whose unsuitability derives from general causes, and not from the condition of the local disease-the very young, the very old, the very feeble, the diabetic, those with very high blood pressure, and, of course, those who absolutely refuse operative treatment. Here, too, I think our course is clear. Interstitial radium treatment carried out on a comprehensive scale unquestionably offers these patients their best chance of long survival.

Then we come to the cases where radical operation is unsuitable owing to the extent of the primary disease, or to gross involvement of the axilla, or even slight involvement of the supraclavicular glands. Here I believe the best course to be a strictly limited operative removal of the tumour and surrounding breast tissue, followed by implantation of radium needles in the axilla and the usual lymphatic paths. One cannot but feel that by removing the major portion of the disease one has put the patient's tissues in a better position to take advantage of the effects of the subsequent radiation therapy. This limited amputation has also proved of great value in my hands in cases where ulceration of the tumour is imminent, or has already become established.

Lastly, we come to the class in which the local condition is more widespread, and where skin nodules, peau d'orange, diffuse invasion of the breast, or involvement of both organs is manifest. In these, again, our course is quite clear. They are naturally quite unsuitable for surgery, and the disease is too extensive for the rather limited powers of radium-I mean, of course, limited in the geographical sense. Deep therapy is the method of choice in such cases as these, with the proviso that the voltage should be as high as possible, and the beam should be delivered with all the intensity that the tissues will stand.

It sounds somewhat paradoxical, but it seems to me that it is in some of the most widespread, and therefore by ordinary standards our most hopeless cases, that the most brilliant results of $x$-ray therapy have been obtained. Their lives are not saved, but they are prolonged in a high degree of comfort for very long periods. It is certain that my $x$-ray colleagues have taught me that we must never accept final defeat in these cases of advanced breast cancer until visceral metastases afford unmistakable evidence that the end is near.

The Northcote Trust, which deals largely with the social supervision of the patients at St. Thomas's Hospital, has completed its twenty-seventh year of work, and its last report covers the twelve months ended October, 1936. It records a real improvement in housing conditions and unemployment, and illustrates the work which the almoners undertake. Help is forthcoming in necessitous cases in ways such as the obtaining of employment, the provision of convalescence treatment, the after-care of cancer cases, and nursing at home. The Northcote Trust Hostel for Girls has been rebuilt, and since its reopening last August has had a full complement of girls and infants. Patients visited at home during the twelve months under review numbered $333 ; 898$ were assisted in other way than by the provision of convalescence facilities or surgical appliances; 993 received convalescent treatment; and 787 surgical instruments were supplied. The income of the Trust during the year was $£ 2,810$, and there was a small credit balance at its close.

\section{VARIATIONS OF WEIGHT DURING PREGNANCY ${ }^{\star}$}

\author{
BY
}

M. D. ARWYN EVANS, M.D., F.R.C.S.Ed.

First Assistant to the Professor of Obstetrics and Gynaecology, Welsh National School of Medicine; Honorary Assistant Gynaecologist to Cardiff Royal Infirmary

The importance of weight-taking during pregnancy and the significance of an abnormal increase has attracted very little attention in this country.

In 1862 Gassner showed that there was a progressive increase in weight of the mother which was greater than that due to the foetus and the hypertrophied reproductive organs. He found that during the last three months of pregnancy there was an average increase of 3.5 to 5.5 pounds. Zangemeister (1916) advocated weight-taking as a means of detecting incipient toxaemia, and in 1925 he showed that during the few days immediately preceding labour there was a definite loss in weight. Hirsch (1922) demonstrated a loss of weight a few days before labour, and ascribed this decrease to the activity of the hypophysis.

Davis (1923) found that the usual gain during pregnancy was 20 pounds. He came to the conclusion that weighttaking should be a routine practice in ante-natal work, since it gave information about latent dropsy. In the same year Hannah found an average gain of 12 pounds during pregnancy. Wieloch (1924) stated that the tendency towards insidious oedema could be detected by systematic weighing of the patient. Randall (1925) found that the average gain in primiparae was 23.2 pounds, and in multiparae 21 pounds. He concluded that the extent of the increase in weight should aid materially in foretelling the toxaemias of later pregnancy.

Slemons and Fagan (1927) found the average gain to be 16.5 pounds. Toombs (1931) made comparisons between the mother's gain during pregnancy and the infant's weight, but failed to establish any relation. Bingham (1932) stated that abnormal weight increase was a precursor of toxaemia, and that it also tended to make the labour more difficult owing to the deposit of fat in the pelvis. He found that the child tended to be larger in proportion to the increase in weight of the mother.

Siddall and Mack (1933) reported that the total gain during the last four months of pregnancy was 15.7 pounds, and concluded that excessive weight gain appeared to be of question. able value in the early recognition of impending toxaemia. Cummings (1934) found the total gain in the first trimester of pregnancy to be less than half a pound, in the second trimester 13 pounds, and in the third trimester 11 pounds. The greatest gain occurred during the sixth and seventh months, in which it was 5 pounds. He stated that an abnormal gain in weight was a very early sign of the toxaemias of pregnancy. During the same year Harding and Van Wyck came to the same conclusion, and therefore advised the practice of weight-taking in pre-natal care.

The present investigation was undertaken to determine the weight increase in normal and toxaemic cases in an attempt to establish standards and to ascertain what significance could be attached to an abnormal gain.

\section{Method of Investigation}

Each patient was examined during the last few months of her pregnancy, weekly, fortnightly, or monthly, depending on the findings at the previous examination. A history of any previous illnesses and pregnancies was taken at the first visit, together with a history of the present pregnancy. A general systematic and obstetrical examination was also made; the patient was weighed, the blood pressure taken, and an examination of the urine performed.

\footnotetext{
* Investigation undertaken during the tenure of a British Medical
} Association Research Scholarship. 